\title{
Effect of Low Pressures on the Room Temperature Transitions of Polytetrafluoroethylene
}

\author{
G. M. Martin and R. K. Eby \\ Institute for Materials Research, National Bureau of Standards, Washington, D.C. 20234
}

(May 24, 1968)

\begin{abstract}
Between approximately 20 and $30{ }^{\circ} \mathrm{C}$ at atmospheric pressure, polytetrafluoroethylene exhibits a phase (denoted as IV), which has not been observed in $P-V-T$ measurements at pressures above $10^{8} \mathrm{Nm}^{-2}$ (1 Kilobar). Data are presented to resolve this phase in the temperature range 0 to $50{ }^{\circ} \mathrm{C}$ and the pressure range 0 to $0.686 \times 10^{8} \mathrm{Nm}^{-2}$. The II-IV $\left(20^{\circ} \mathrm{C}\right)$ transition pressure increases with temperature according to the equation $P=\left(-820+26.9 t+0.68 t^{2}\right) \times 10^{5}$ and the $\mathrm{IV}-\mathrm{I}\left(30^{\circ} \mathrm{C}\right)$ transition according to the equation $P=\left(-91-57.8 t+1.99 t^{2}\right) \times 10^{5}$ suggesting a possible triple point near $2.6 \times 10^{8} \mathrm{Nm}^{-2}$ and $54{ }^{\circ} \mathrm{C}$. However, the IV -1 transition probably cannot be resolved by volume measurements at pressures above $2 \times 10^{8} \mathrm{Nm}^{-2}$ because its volume of transition is small and the two transitions overlap. The enthalpies of the transitions are calculated for different pressures and some thermodynamic properties of phase IV are determined indirectly. It is shown that, if the effect of volume change is subtracted, random reversals of the hand of the helical conformation of the molecule can account for appreciable fractions of the enthalpy and corresponding entropy change for the combined transitions at atmospheric pressure.
\end{abstract}

Key Words: Dilatometry; enthalpy; entropy; helical molecule; low pressure; phase diagram; polytetrafluoroethylene; transitions; triple point.

Volume measurements over a wide range of temperature and pressure have established a phase diagram for polytetrafluoroethylene with three solid phases and the melt $[1,2,3,4] .{ }^{1}$ Most of the data were obtained by the piston displacement method and had a low pressure limit of about $10^{8} \mathrm{Nm}^{-2}$ (1 Kilobar) imposed by friction. They show a transition line which extrapolates to the " $20{ }^{\circ} \mathrm{C}$ " transition observed in measurements at atmospheric pressure but do not show a separate line extrapolating to the smaller " $30{ }^{\circ} \mathrm{C}$ " transition which is also observed at atmospherical pressure $[5,6,7]$. This may be due to a lack of sensitivity in the method, but it is also possible that the " $30{ }^{\circ} \mathrm{C}$ " transition line terminates in a triple point with the " $20^{\circ} \mathrm{C}$ " transition line at some pressure below $10^{8} \mathrm{Nm}^{-2}$. Low pressure data of sufficient accuracy to determine this have not been available. This note reports measurements obtained with a dilatometer at temperatures between 0 and $50{ }^{\circ} \mathrm{C}$ and pressures up to $0.686 \times 10^{8} \mathrm{Nm}^{-2}$.

The polytetrafluoroethylene was similar to a sample used in other measurements [8] (sample number 3). At the start of the present work, the density determined at $25{ }^{\circ} \mathrm{C}$ by hydrostatic weighing was $2.253 \mathrm{~g} / \mathrm{cm}^{3}$

\footnotetext{
' Figures in brackets indicate the literature references at the end of this paper.
}

which corresponds to a crystallinity of about 90 percent. The data given here are based on the sample as a whole and have not been subjected to the small correction for crystallinity.

The polymer was evacuated for $16 \mathrm{hr}$ at a pressure of $1.3 \times 10^{-3} \mathrm{Nm}^{-2}\left(10^{-5}\right.$ torr $)$ in a glass dilatometer, which was then filled with mercury. The dilatometer was placed inside a pressure vessel which was immersed in a temperature bath regulated within \pm 0.05 ${ }^{\circ} \mathrm{C}$. Windows in the bath and in the pressure vessel permitted measurement of the mercury level with a cathetometer. An estimated uncertainty of $\pm 5 \times 10^{-3}$ $\mathrm{cm}$ in this reading corresponds to an uncertainty of less than $\pm 2 \times 10^{-5} \mathrm{~cm}^{3} / \mathrm{g}$ in the calculated specific volume. Pressure was measured with a calibrated Bourdon type gage (uncertainty less than $\pm 10^{5} \mathrm{Nm}^{-2}$ ). Observed volume changes were corrected for changes in the volume of the glass and mercury. Details of the apparatus have been described previously [9].

Volume measurements were made as a function of temperature at each pressure. The pressure was applied at $50{ }^{\circ} \mathrm{C}$ and the temperature lowered in steps at the rate of about one degree per hour, which was decreased to $1 / 4$ to $1 / 24$ degree per hour near the " $20{ }^{\circ} \mathrm{C}$ " transition. After the lowest temperature was reached, the sample was heated in the same manner to $50{ }^{\circ} \mathrm{C}$ and the pressure released. 
Figure 1 shows the specific volume as a function of temperature for each pressure in the range of the transitions. Equilibrium volumes are given except at the $20{ }^{\circ} \mathrm{C}$ transition, where the volume at a given temperature changes slowly over a long period of time and in some cases was still varying linearly with the logarithm of time after $24 \mathrm{hr}$. The $20{ }^{\circ} \mathrm{C}$ transition takes place about $1.6^{\circ} \mathrm{C}$ lower on cooling than on heating [6] and this difference is approximately independent of pressure. The $30{ }^{\circ} \mathrm{C}$ transition did not exhibit hysteresis and the data show that it can be observed even at the highest pressure, $0.686 \times 10^{8} \mathrm{Nm}^{-2}$.

Figure 2 shows the phase diagram at low pressure. Transition temperatures are taken to be the temperatures of the maximum slopes in the specific volumetemperature curves since these values could be determined more precisely than the points at which the last trace of the low temperature phase disappears. The intermediate phase is called IV to distinguish it from those already numbered [1]. The temperature difference between the two transitions decreases with increasing pressure and extrapolations based on the second degree curves shown in figure 2 indicate a possible triple point at approximately $2.6 \times 10^{8}$ $\mathrm{Nm}^{-2}$ and $54{ }^{\circ} \mathrm{C}$. Such a triple point is out of the range of the present apparatus and in the range of the usual high pressure techniques. However, as discussed later, there is some doubt whether volume measurements will be able to resolve the IV-I transition at higher pressures.

Straight lines were fitted to the specific volumetemperature data below the II-IV and above the IV-I transitions and the total volume change for both transitions was found by taking the difference between

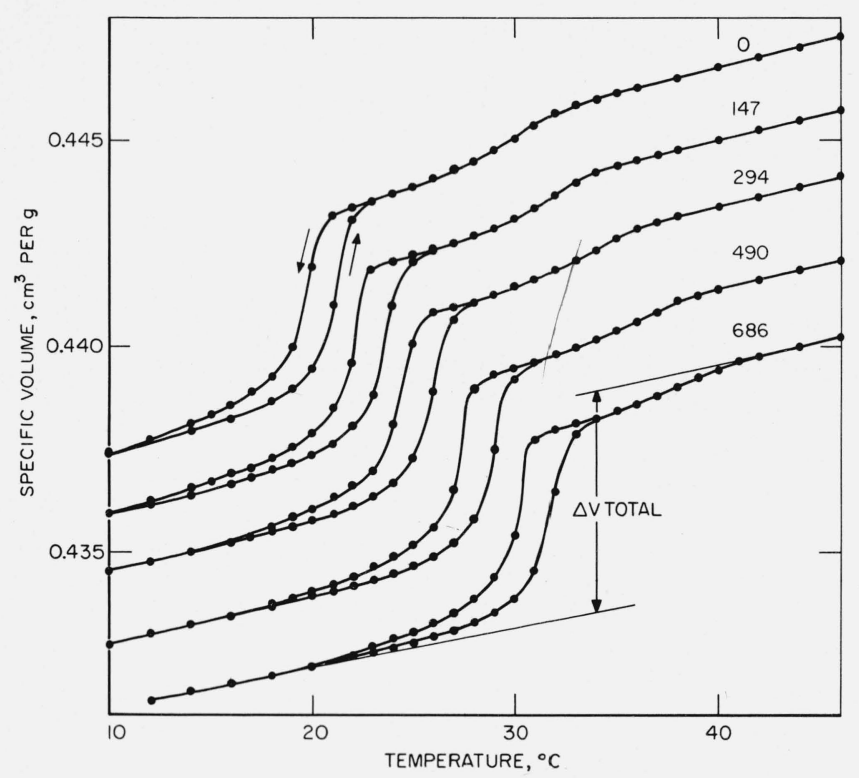

FIGURE 1. Specific volume versus temperature for polytetrafluoroethylene in the region near the room temperature transitions. The numbers on the curves give the gage pressures in $10^{5} \mathrm{Nm}^{-2}$. these lines (see fig. 1). This technique was not as satisfactory for determining the volume changes of each transition separately, because the two transitions overlap. Therefore, plots of the slopes of the specific volume-temperature curves against temperature (fig. 3) were used for the analysis. The shaded areas were measured to obtain the IV-I transition volumes and these values could then be subtracted from the total volume changes to find the II-IV transition volumes. This method is based on the approximation that the cutoff "tails" of each transition have equal areas. Although other assumptions could be made, they could not be shown to yield more accurate results.

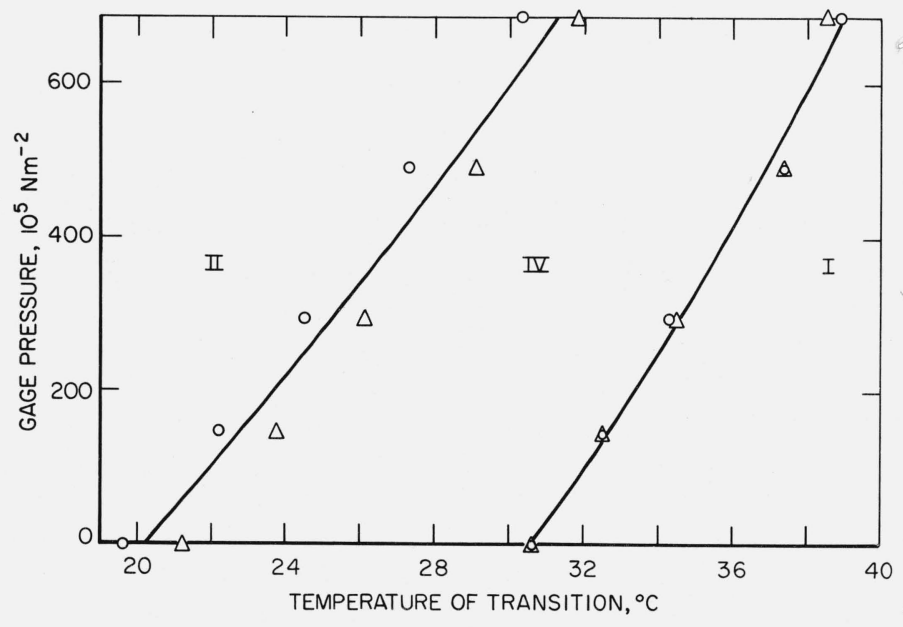

FIGURE 2. Relations between pressure and transition temperatures for polytetrafluoroethylene.

The lines represent the second degree curves $P=\left(-820+26.9 t+0.68 t^{2}\right) \times 10^{5}$ (II-IV) and $P=\left(-91-57.8 t+1.99 t^{2}\right) \times 10^{5}$ (IV-I) which were fitted to the data, $\mathrm{O}=$ cooling; $\Delta=$ heating.

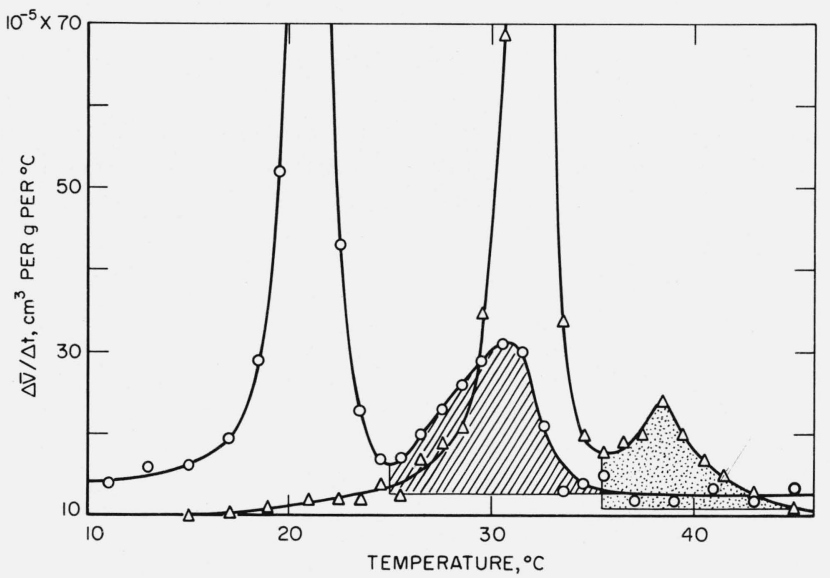

Figure 3. Slopes of specific volume versus temperature curves plotted against temperature for polytetrafluoroethylene. Heating cycle. $\bigcirc=0 \times 10^{5} \mathrm{Nm}^{-2}$ pressure; $\Delta=686 \times 10^{5} \mathrm{Nm}^{-2}$ pressure. 
Figure 4 shows that, while the total transition volume and the IV-I transition volume decrease, the II-IV transition volume does not vary significantly with increasing pressure. Less accurate measurements made directly on the specific volume-temperature curves in the same way that the total transition volumes were determined yielded slightly smaller II-IV transition volumes, but did confirm the lack of variation with pressure. Because of the small transition volume at $0.686 \times 10^{8} \mathrm{Nm}^{-2}$, the decreasing trend in this quantity, and the decreasing temperature separation of the two transitions, the IV-I transition would be difficult to resolve by volume measurements at pressures above $2 \times 10^{8} \mathrm{Nm}^{-2}$. Probably a technique such as $\mathrm{x}$-ray diffraction would be required.

The Clapeyron equation may be written

$$
\Delta H=T \Delta V d P / d T
$$

In it, $\Delta H, T, \Delta V$, and $P$ represent the heat, absolute temperature, volume, and pressure of transition, respectively. Values of $\Delta H$ for the II-IV and IV-I transitions at one atmosphere calculated by eq (1) are in reasonable agreement with data in the literature $[4,7,10,11]$ and with results obtained by differential scanning calorimetry on the present sample.

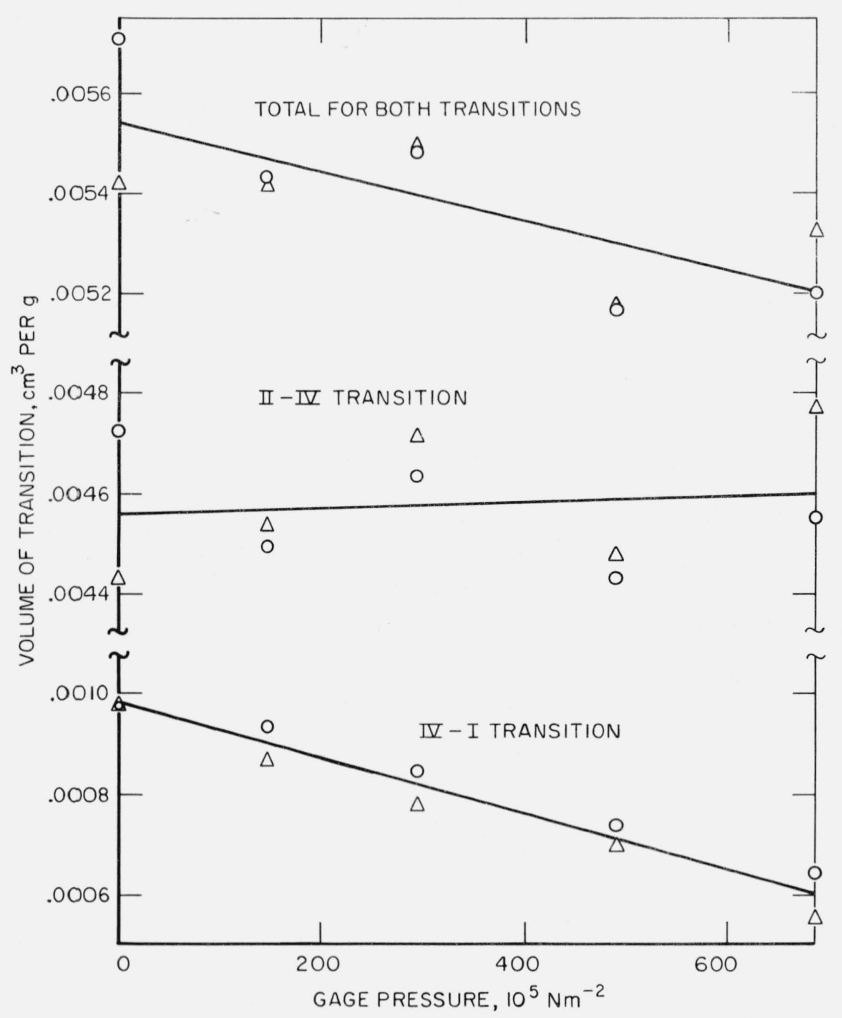

FIGURE 4. Change in volumes of transition with pressure for poly. tetrafluoroethylene.

$O=$ cooling; $\Delta=$ heating. The straight lines are fitted to the data by least squares.
(The D.S.C. results were obtained at much greater rates of heating and cooling and varied appreciably from measurement to measurement.) The transition heats were also calculated for other pressures and are given in figure 5 . For the II-IV transition, $\Delta H$ increases with pressure while for the IV-I it is almost unchanged.

The following equations also apply to the transitions:

$$
d \Delta V / d P=(d T / d P) \Delta \alpha+\Delta \beta
$$

and

$$
d \Delta H / d P=(d T / d P) \Delta C_{P}+\Delta V-T \Delta \alpha .
$$

In these, $\alpha \equiv(\partial V / \partial T)_{P}, \beta \equiv(\partial V / \partial P)_{T}$, and $C_{P} \equiv(\partial H /$ $\partial T)_{P}$. Now, the modulus for ultrasonic longitudinal waves is the same in phases IV and II [8]. This suggests that $\beta_{\mathrm{IV}}$ might be approximately equal to $\beta_{\mathrm{II}}$. If it is assumed that this is correct, the data in figures $1,2,4$, and 5, together with eqs (2) and (3), yield the following approximate values for phase IV at one atmosphere: $\alpha_{\mathrm{II}}=\alpha_{\mathrm{IV}}$ and $\mathrm{C}_{P_{\mathrm{I}}}=C_{P_{\mathrm{II}}}$. In this way, the properties of phase IV can be determined even though they cannot be measured directly because of the overlap of the transitions.

Experimental support has been presented for the occurrence in the crystals of randomly located reversals of the pitch of the molecular helix, i.e., a change from a left to a right handed or from a right to a left handed helix [12]. Experimental data yield an energy of reversal of $0.053 \mathrm{eV}$ in Phase $\mathrm{I}$ and calculations based on three models of molecular interaction give an average ratio of the energy of reversal in phase II to that in phase I of 1.16 [12]. On this basis, the energy of reversal in phase II can be taken as $0.062 \mathrm{eV}$. These numbers may be used to calculate the concentration

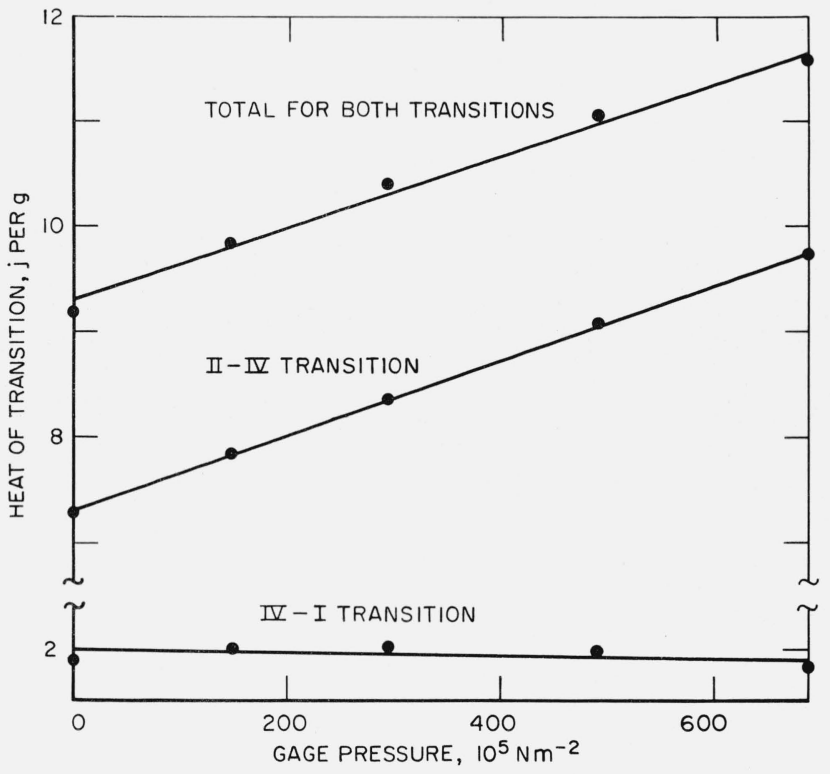

FIGURE 5. Variation of heat of transition with pressure for polytetrafluoroethylene.

The straight lines are fitted to the data by least squares. 
of random reversals present in phase II at the II-IV transition temperature and in phase $\mathrm{I}$ at the IV-I transition temperature and from these the associated changes in entropy and enthalpy. ${ }^{2}$ This can be accomplished by first using the equation for the Boltzmann distribution: $n_{1} / n_{2}=\exp (-E / k T)$ where $n_{1}$ is the number of reversals, $n_{2}$ is the number of bonds not reversed, $E$ the energy of reversal, and $k$ the Boltzmann constant. The entropy of mixing is then given by $s=k \ln \left(n ! / n_{1} ! n_{2} !\right)$ where $n$ is the total number of bonds. The resulting changes in enthalpy and entropy are $2.2 \mathrm{~J} / \mathrm{g}$ and 0.013 $\mathrm{J} / \mathrm{gK}$, respectively. If we assume that these changes occur only at the transitions (neglecting any change within the small temperature range of phase IV), the above values can be compared with the total enthalpy change given in figure 5 and the corresponding entropy change. After correction for the effects of volume change $[4,14]$, the latter values are $7.6 \mathrm{~J} / \mathrm{g}$ and $0.026 \mathrm{j} / \mathrm{gK}$. Thus, randomly located reversals of the hand of the helix can account for about 30 percent of the enthalpy change at constant volume and about 50 percent of the entropy change at constant volume that occurs at the transitions. Of the quantities in the calculations above, the value of $E$ in phase II is probably the least accurate. For example, it corresponds to eight reversals of pitch per 100 bonds, a number not consistent with the $x$-ray data [4]. If $E_{\text {II }}$ were $0.080 \mathrm{eV}$, the calculated enthalpy and entropy would be $5.7 \mathrm{~J} / \mathrm{g}$ and $0.032 \mathrm{~J} / \mathrm{gK}$, respectively. These values give the best fit to the experimental data and correspond to the more reasonable concentration of four reversals per 100 bonds.

In summary, $P V T$ measurements indicate that phase IV of polytetrafluoroethylene probably exists at pres-

2 A similar calculation has been presented recently, but it assumed that there were no reversals in phase II [13]. sures up to $2.6 \times 10^{8} \mathrm{Nm}^{-2}$. The diffuse IV-I transition and the triple point will be difficult to detect with high pressure techniques because of the small transition volume and the small temperature separation from the diffuse II-IV transition. Enthalpies and entropies of transition have been calculated from the $P V T$ data and compared with those calculated for changes in the number of randomly located reversals of the hand of the helical molecule. The results show that such reversals can account for appreciable fractions of the enthalpy and entropy of the combined II-IV and IV-I transitions at atmospheric pressure. The values of $(\delta V / \delta T)_{p}$ and $(\delta V / \delta P)_{T}$ in phase IV are estimated to be approximately the same as in phase II while the value of $(\delta H / \delta T)_{p}$ in phase IV is estimated to be close to that in phase I.

\section{References}

[1] Weir, C. E., J. Res. NBS 50, 95 (1953) RP2395.

[2] Beecroft, R. I., and Swenson, C. A., J. Appl. Phys. 30, 1793 (1959).

[3] Pistorius, C. W. F. T., Polymer 5, 315 (1964).

[4] Sperati, C. A., and Starkweather, H. W., Jr., Fortschr. Hoch Polym.-Forsch. 2, 465 (1961). (In English.)

[5] Rigby, H. A., and Bunn, C. W., Nature 164, 583 (1949).

[6] Quinn, F. A., Jr., Roberts, D. E., and Work, R. N., J. Appl. Phys. 22, 1085 (1951).

[7] Yasuda, T., and Araki, Y., J. Polymer Sci. 5, 331 (1961).

[8] Eby, R. K., and Sinnott, K. M., J. Appl. Phys. 32, 1765 (1961).

[9] Martin, G. M., and Mandelkern, L., J. Appl. Phys. 34, 2312 (1963).

[10] Furukawa, G. T., McCoskey, R. E., and King, G. J., J. Res. NBS 49, 273 (1962) RP2364.

[11] Marx, P., and Dole, M., J. Am. Chem. Soc. 77, 4771 (1955).

[12] Brown, R. G., J. Chem. Phys. 40, 2900 (1964).

[13] Bates, T. W., and Stockmayer, W. H., Macromolecules 1, 17 (1968).

[14] Starkweather, H. W., Jr., and Boyd, R. H., J. Phys. Chem. 64, 410 (1960).

(Paper 72A5-512) 